\title{
La técnica del golpeado, etnografía comparada de procesos de elaboración cerámica en dos comunidades alfareras del austro ecuatoriano: Jatumpamba y Las Nieves
}

La technique du battage, ethnographie comparative des processus d'élaboration de la céramique dans deux communautés potières de la Sierra sud de l'Équateur

Jatumpamba et Las Nieves

The beating technique: comparative ethnography of ceramic manufacturing processes in two potter communities of Southern Ecuador: Jatumpamba and Las Nieves

\section{Mario Brazzero}

\section{(2) OpenEdition}

\section{Edición electrónica}

URL: https://journals.openedition.org/bifea/11699

DOI: $10.4000 /$ bifea.11699

ISSN: 2076-5827

Editor

Institut Français d'Études Andines

\section{Edición impresa}

Fecha de publicación: 1 abril 2020

Paginación: 85-105

ISSN: 0303-7495

\section{Referencia electrónica}

Mario Brazzero, «La técnica del golpeado, etnografía comparada de procesos de elaboración cerámica en dos comunidades alfareras del austro ecuatoriano: Jatumpamba y Las Nieves», Bulletin de l'Institut français d'études andines [En línea], 49 (1) | 2020, Publicado el 08 agosto 2020, consultado el 24 agosto 2021. URL: http://journals.openedition.org/bifea/11699; DOl: https://doi.org/10.4000/ bifea. 11699

\section{(c)}

Les contenus du Bulletin de l'Institut français d'études andines sont mis à disposition selon les termes de la licence Creative Commons Attribution - Pas d'Utilisation Commerciale - Pas de Modification 4.0 International. 


\title{
La técnica del golpeado, etnografía comparada de procesos de elaboración cerámica en dos comunidades alfareras del austro ecuatoriano: Jatumpamba y Las Nieves
}

\author{
Mario Brazzero*
}

\section{Resumen}

Los estudios arqueológicos sobre el austro ecuatoriano han sustentado su análisis en la forma y diseño de la cerámica para constituir cronologías y relaciones interregionales, prestándoles poca atención a los aspectos técnicos y a las herramientas utilizadas en los procesos de elaboración. Este estudio, de carácter etnográfico, registra las técnicas empleadas en dos comunidades alfareras de la Sierra Sur del país — Jatumpamba y Las Nieves_ que comparten el uso de golpeadores o huactanas, herramientas de posible origen prehispánico. Los resultados del análisis comparativo entre estas localidades pretenden abonar al reconocimiento de la necesidad de generar líneas de investigación sobre la tecnología cerámica, tanto en contextos arqueológicos como etnográficos.

Palabras clave: tecnología cerámica, Tacalshapa, Jatumpamba, Las Nieves

La technique du battage, ethnographie comparative des processus d'élaboration de la céramique dans deux communautés potières de la Sierra sud de l'Équateur : Jatumpamba et Las Nieves

\section{Résumé}

Les études archéologiques sur le sud de l'Équateur ont basé leurs analyses sur la forme et les décors des céramiques pour constituer des chronologies et établir des interactions régionales, tout en accordant

Universidad del Azuay, docente-investigador. Master en Antropología Visual y Documental Antropológico. Facultad Latinoamericana de Ciencias Sociales (FLACSOEcuador). E-mail: mbrazzero@gmail.com 
peu d'attention aux aspects techniques et aux outils utilisés dans les processus de fabrication. Cette étude ethnographique rend compte des techniques utilisées dans deux communautés de potiers de la Sierra sud du pays - Jatumpamba et Las Nieves - , ont en commun I'utilisation de battoirs ou huactanas, outils sans doute d'origine préhispanique. Les résultats de l'analyse comparative entre ces sites montrent la nécessité de proposer des axes de recherche sur la technologie céramique, aussi bien dans des contextes archéologiques qu'ethnographiques.

Mots-clés : technologie céramique, Tacalshapa, Jatumpamba, Las Nieves

\title{
The beating technique: comparative ethnography of ceramic manufacturing processes in two potter communities of Southern Ecuador: Jatumpamba and Las Nieves
}

\begin{abstract}
The archeological studies of ceramics in Southern Ecuador have based their analyses on shapes and designs in order to generate chronologies and interregional relationships, paying less attention to the technical aspects and the tools that have been used in the manufacturing process. This ethnographic study registered the techniques used in two Southern highlands communities — Jatumpamba and Las Nieves - which share the use of beaters or huactanas, tools of possible pre-hispanic origin. The results of the comparative analysis of these two locations, demonstrates the need to generate new lines of research on ceramic technology, both in archaeological and ethnographic contexts.
\end{abstract}

Keywords: Ceramic technology, Tacalshapa, Jatumpamba, Las Nieves

\section{INTRODUCCIÓN}

Este estudio es el resultado del proyecto denominado «Investigación del Patrimonio Cultural Inmaterial vinculado a la alfarería con técnicas prehispánicas de Jatumpamba y Las Nieves», localidades rurales de las provincias australes del Cañar y Azuay respectivamente (fig. 1), realizado por iniciativa del Instituto Nacional de Patrimonio Cultural (INPC) Regional 6, en el año 2011. El objetivo consistía en «investigar y registrar el Patrimonio Cultural Inmaterial asociado a la alfarería tradicional de Jatumpamba y las Nieves», con la finalidad de realizar un diagnóstico que proponga medidas para su salvaguarda.

Las huactanas o golpeadores son herramientas de barro cocido «conformadas por un mango de prensión de forma cilíndrica, apegado a la parte activa, hemisférica» (Lara, 2017: 98). Se las usa principalmente para formar y alisar las vasijas durante su elaboración. En las zonas de estudio son de dos tipos: uno cóncavo para trabajar las paredes externas, y otro convexo para trabajar el interior de la vasija (fig. 2). Sus dimensiones pueden variar en función del porte del ceramio en preparación. Para una huactana mediana, la altura se ubica en los rangos de 7 a 9 cm, con un mango de entre 4 y 4,5 cm de diámetro y un disco de 7 a $10 \mathrm{~cm}$ de diámetro, siendo los discos de los golpeadores cóncavos algo más grandes que los convexos; el peso varía entre 350 y 450 gramos (las formas, dimensiones y peso están descritos de manera más detallada en: Lara, 2017: anexo 7). El uso de estos artefactos es 


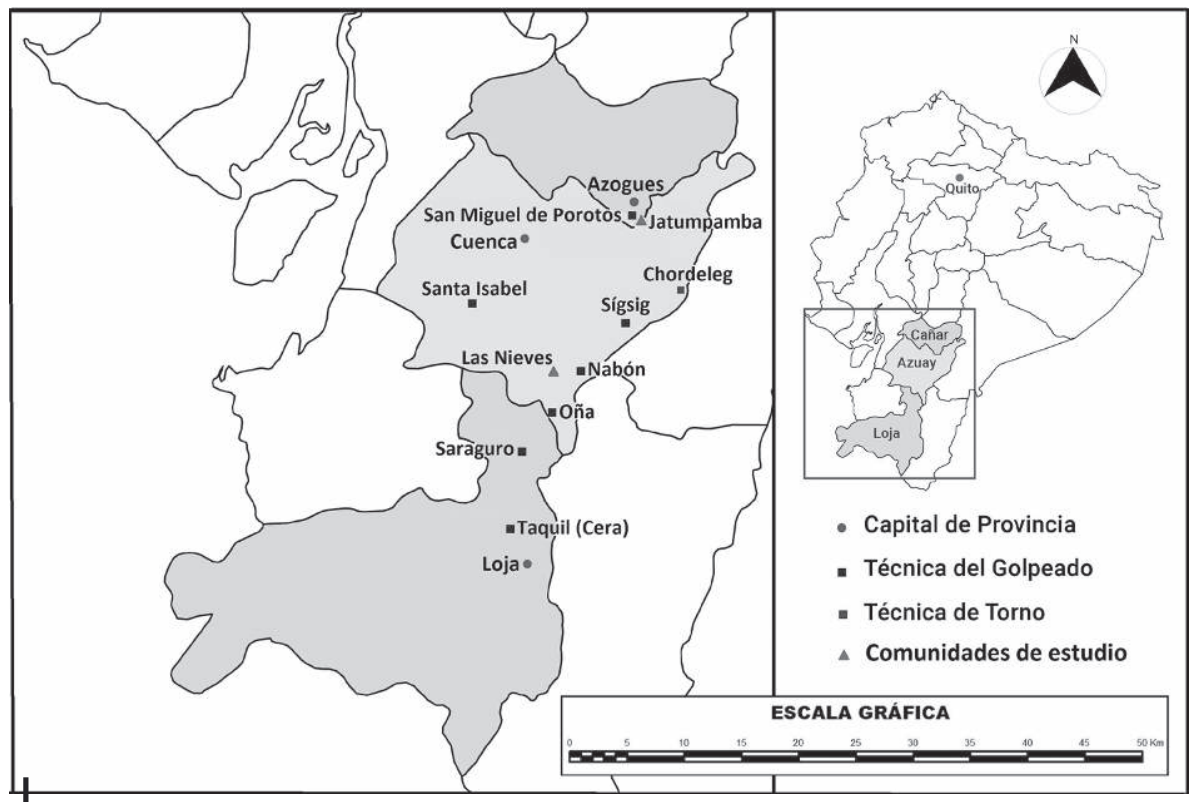

Figura 1 - Ubicación de Jatumpamba y Las Nieves dentro del territorio que reporta la técnica del golpeado

(C) M. Brazzero, 2020

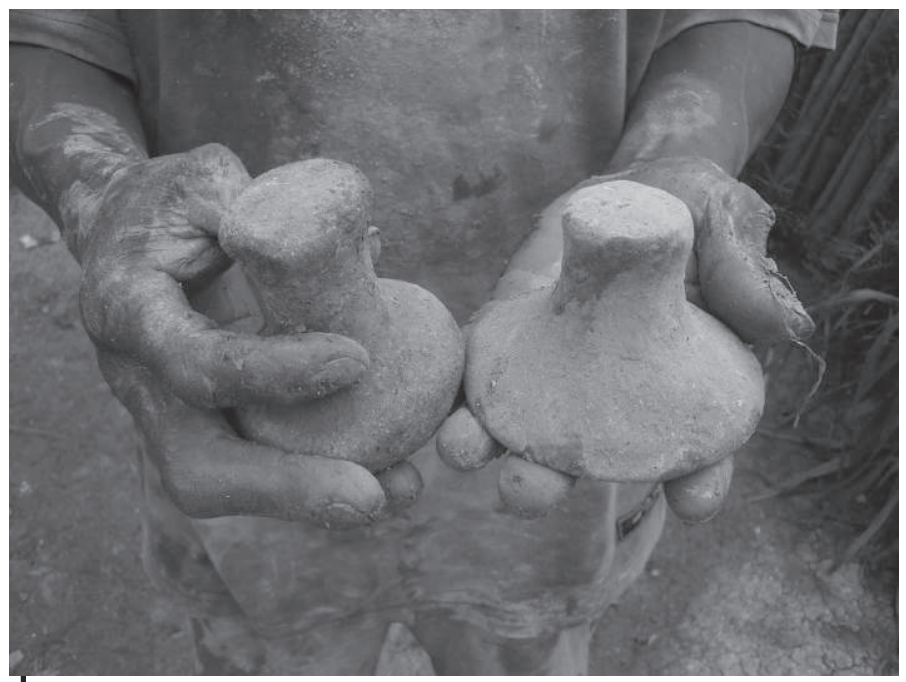

Figura 2 - Golpeadores o huactanas de Jatumpamba

(C) M. Brazzero, 2011

reportado en varios sectores de la Sierra Sur del Ecuador, entre ellos Jatumpamba, Olleros y Zhorzhán en la provincia del Cañar; Las Nieves, Santa Isabel, Oña y Sígsig en Azuay; Saraguro y Taquil en Loja (fig. 1), lo que convierte la técnica del golpeado con el uso de huactanas en una de las más importantes de esta región. 
La comunidad de Jatumpamba pertenece a la parroquia San Miguel de Porotos del cantón Azogues. Está integrada por 3 parcialidades: Jatumpamba, Pacchapamba y Chico Ingapirca (fig. 1: San Miguel de Porotos). Esta comunidad, creada en el año 1952, está localizada a 11 km al sur de Azogues, capital de la provincia del Cañar.

La parroquia Las Nieves pertenece al cantón Nabón de la provincia del Azuay (fig. 1). La conforman 12 comunidades, con una población aproximada de 450 familias. Está localizada a $60 \mathrm{~km}$ al sur de Cuenca, en la vía que lleva a la provincia de Loja.

El tiempo establecido por el INPC para la investigación fue de cuatro meses, por lo que debía planificar una logística que me permita la revisión bibliográfica de la arqueología local, con énfasis en investigaciones sobre las tecnologías cerámicas y el trabajo de campo con los alfareros y alfareras de Jatumpamba y Las Nieves.

\section{CONOCIMIENTOS ACTUALES SOBRE LA CERÁMICA ARQUEOLÓGICA DE LA SIERRA SUR DEL ECUADOR}

En los años 20 del siglo pasado, Max Uhle inició estudios en el sitio de Cerro Narrío, ubicado en la actual ciudad de Cañar y correspondiente al periodo Formativo medio y tardío (1500 - 500 a. C.). Basó su análisis en las «analogías estilísticas que encontró en las producciones cerámicas e iconográficas de la América andina (fig. 3a), apoyado por el estudio de 'estratigrafía cultural'» (Gomis, 2019: 35).

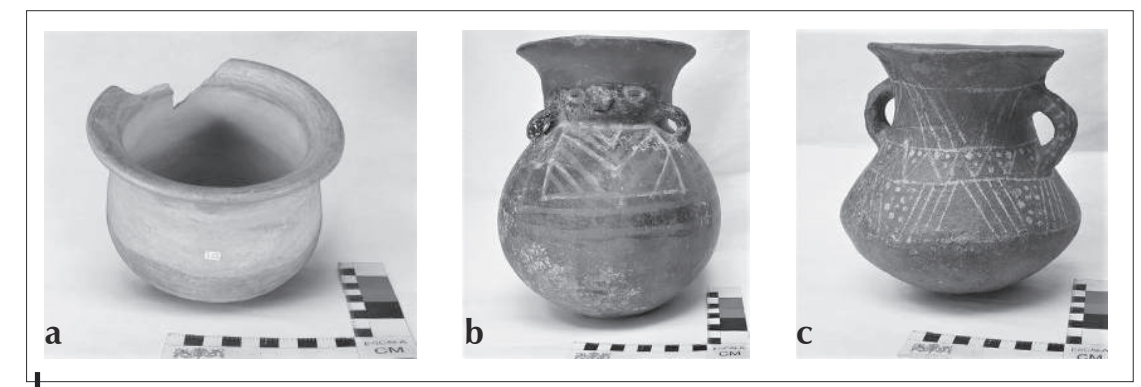

Figura 3 - a: recipiente Narrío (1M2-2998-86). b: recipiente Tacalshapa (1M2-3748-86). c: recipiente Cashaloma (1M2-6592-86)

(C) Museo Remigio Crespo Toral, Cuenca

Siguiendo el modelo de Uhle, se establecieron semejanzas que permitieron hablar de un centro de contacto interregional desde tiempos muy tempranos (Collier \& Murra, 2007 [1943]: 72-93), o un complejo cerámico homogéneo que correspondería a la fase tardía del Formativo de la Sierra Sur del Ecuador, con relaciones y contactos tanto con las culturas de la costa y oriente ecuatorianos como con las de los Andes Centrales, hoy Perú (Gomis, 2007).

Para el caso de Tacalshapa, Jaime Idrovo (2000: 52-58), identifica cronológicamente 3 periodos: Tacalshapa I (500 a. C.-200/100 a. C.), Tacalshapa II (200/100 a. C.- 
500 d. C.) y Tacalshapa III (500 d. C.-1100/1200 d. C.), lo que permite establecer la existencia de probables relaciones interculturales entre Tacalshapa I y Narrío y Chorrera (esta última ubicada en la costa ecuatoriana), entre Tacalshapa II (fig. 3b) y Moche y Vicús (Costa Norte del Perú) y entre Tacalshapa III y la cerámica que se produce en Jatumpamba (comunicación personal, 2011). El «engobe rojo simple» concuerda con el de la cerámica etnográfica de Jatumpamba, pero la decoración «Con líneas rojas sobre color natural» estaría más cerca de la que se realiza en la cerámica de Las Nieves, según pude constatar en el campo.

A diferencia de Idrovo, Albert Meyers (2011 [1998]: 172-195) propuso cuatro fases cronológicas para Tacalshapa, y estableció elementos comunes entre las piezas de su fase Tacalshapa II (que provienen de los valles de Cañar, Cuenca, Azogues y Taday) y los estilos cerámicos Gallinazo y Puerto Moorin del norte del Perú, así como entre las botellas de Tacalshapa IV y las últimas fases del estilo Moche.

El estilo Cashaloma (periodo de Integración: 1000 d. C. hasta la conquista inkaica); se concentra mayoritariamente en el valle de Cañar (fig. 3c), con escasos reportes en el valle de Cuenca-Azogues, donde es más frecuente el estilo Guapondelig. Puede estar presente también en Jatumpamba, confundido con Tacalshapa III, aunque con ciertas diferencias (Idrovo, comunicación personal, 2011).

De manera general, estos trabajos arqueológicos mantuvieron la comparación de las características visuales de la cerámica como sello característico de su producción intelectual y, en su mayoría, dejaron de lado las tecnologías cerámicas, tanto arqueológicas como etnográficas.

\section{EL GOLPEADO, ¿TÉCNICA PREHISPÁNICA?}

De lo poco que existe en la literatura arqueológica ecuatoriana sobre las tecnologías cerámicas en la zona de estudio, son de resaltar los trabajos de Holm, quien se interesa en un grupo de golpeadores de una colección de «objetos de barro» del Museo Salesiano de Cuenca, clasificados como moledores pero no descritos anteriormente en la literatura arqueológica ni etnográfica, y sin asociación a contextos arqueológicos específicos. Un aporte del trabajo de Holm radica en que, durante sus visitas a las alfareras de Jatumpamba, no solo describió las tecnologías cerámicas, sino que, en colaboración con especialistas en el ramo, sometió a análisis químicos los fragmentos de cerámica, arcilla y desgrasante (Holm, 1961: 163). Al hablar de la temporalidad de la técnica, Holm concluye que:

Al aceptarse estas pruebas, muy insuficientes, por cierto, tendríamos la profundidad temporal que requiere la presencia actual de esta técnica alfarera en el Austro, pero tenemos que reconocer que nos faltaría por completo la transición técnica entre la pre-historia y la actualidad, porque la cerámica española constituye todavía una gran incógnita en los estudios ecuatorianos (Holm, 1961: 201).

Sjöman (1991: 71) advierte la posibilidad del origen de esta técnica en los pueblos cañaris asociados a la cerámica Tacalshapa, además de la inca, ambas presentes 
en San Miguel de Porotos. Sin embargo, reconoce que esta información resulta de «recolección en superficie» sobre la que no brinda mayor información, ya que, según la autora, no han existido excavaciones científicas en la zona. Idrovo establece que el origen y difusión de la técnica estaría asociado a la cultura material cañari. Asegura que la técnica del golpeado y las huactanas se utilizaron en la cerámica Tacalshapa, aunque no especifica los argumentos que lo llevan a esta afirmación, siendo esta el resultado de «la revisión de numerosas piezas Tacalshapa del Museo del Banco Central de Cuenca» (Idrovo, comunicación personal, 2011). Sustenta además su hipótesis en el hallazgo de una huactana «de uso alfarero a partir de Tacalshapa Il», recuperada en una terraza de Pumapungo y asociada a un horno de cerámica (Idrovo, 2000: 192). Si bien Sjöman (1991: 71) asegura que los golpeadores se encuentran en diferentes contextos arqueológicos en la provincia del Azuay, no especifica cuáles son estos, por lo que la huactana reportada por Idrovo sería la única registrada en un contexto arqueológico, de lo que conozco.

Otra evidencia que podría reforzar la hipótesis sobre la antigüedad de la técnica es, según Idrovo, el sistema de cocción, ya que, en su opinión, el tipo de hornos que se encuentran en las inmediaciones de Jatumpamba coinciden exactamente con el único horno encontrado en contexto arqueológico en Pumapungo (comunicación personal, 2011).

Sin embargo, después de inspeccionar el posible horno de Pumapungo y los hornos de la comunidad de Olleros, vecina de Jatumpamba, no encontré aquellas coincidencias exactas de las que habla Idrovo. Estas están relacionadas con el material de construcción de los dos hornos que es la piedra, la altura de las paredes y, en menor medida, con su espacio interior, aunque aquel de los hornos de Olleros sea más amplio. Pero existe una diferencia sustancial en su estructura: el horno de Pumapungo no tiene las aberturas posteriores para el ingreso del oxígeno que tienen los hornos de la comunidad de Olleros, indispensables para la circulación del aire que incremente el calor interior, según me explicaron los artesanos de la zona (figs. 4 y 5).

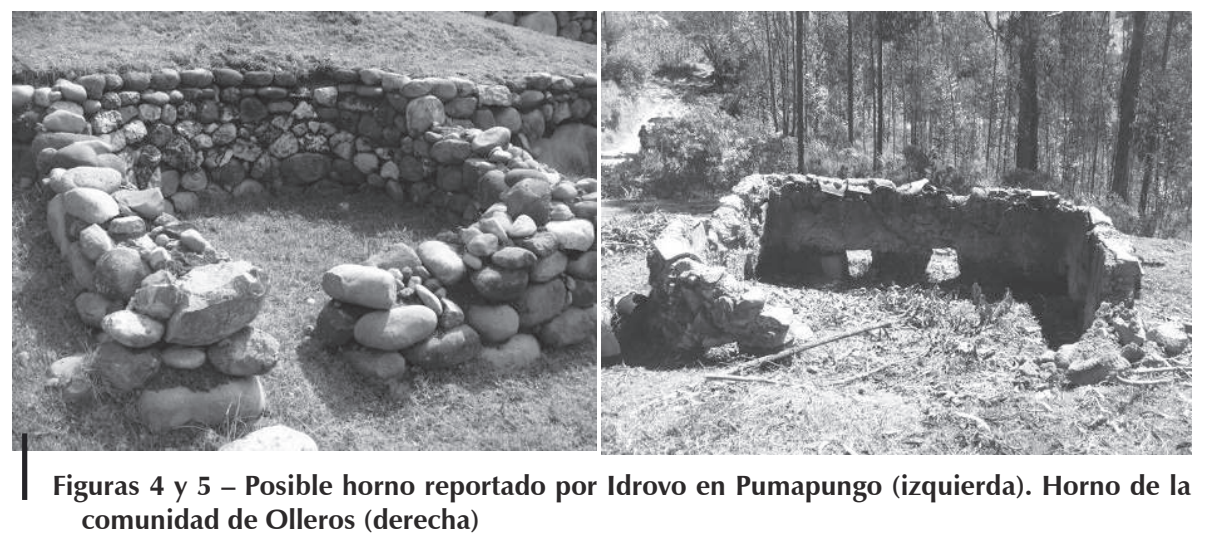

(C) M. Brazzero, 2011 
Catherine Lara (2019) dio un paso importante al aplicar el enfoque tecnológico para identificar la cadena operativa (transformación de la arcilla en cerámica) de 117 objetos Tacalshapa y Cashaloma y 771 fragmentos excavados por la autora en el valle del río Cuyes (provincia de Morona Santiago). Lara identificó, por medio del análisis macro y microscópico de las muestras, un grupo técnico que presentaba huellas de golpeado sobre la base y el cuerpo (Lara, 2019: 189). Este grupo técnico estaría conformado por cerámica Tacalshapa II de la clasificación de Idrovo, pero también por Tacalshapa I y, en menor medida, por cerámica Cashaloma (Lara, 2019: 193). Este hallazgo científico probaría la continuidad del uso de golpeadores hasta la actualidad (ver también Lara, este volumen).

En la Colonia, la región de Azogues fue reportada como productora de cerámica por fray Gaspar de Gallegos, en la Relación Geográfica de Peleusí de Azogues de 1582, cuando escribe:

Hay en este pueblo muy buen barro para loza, y hácese respecto desto mucha loza, así de tinajas, jarros y ollas y cántaros y otras vasijas para el servicio de los españoles y naturales. Es una loza muy colorada que se tiene en mucho, y así están los olleros aquí de muy antiguos tiempos, que desde el tiempo del Inga hay muy buenos oficiales deste oficio, aunque no son tan naturales, sino traspuestos aquí por respecto del buen aparejo que hay para la dicha loza. Y hay mucho bolarménico con que estos indios hacen la loza muy colorada con un matiz ques casi como de vidrio, que le dan con el dicho bolarménico. Tiénese en mucho esta loza y es muy nombrada en todo este distrito (Gallegos, 1897 [1582]: 28).

Esta cita ha sido interpretada por varios autores y con diferentes enfoques (Holm, 1961; Idrovo, 1990; Sjöman, 1992; Arteaga, 2000). Olaf Holm asegura que Peleusí de Azogues incluye a los centros alfareros que aún existen en la parroquia de San Miguel de Porotos (Jatumpamba, Zhorzhán y Olleros: figura 1), y que, debido a que en aquella época la parroquia no había iniciado su vida eclesiástica o civil, no había razón para que sea nombrada por el clérigo (Holm, 1961: 170). Idrovo sostiene que la Relación se refiere a la zona de Jatumpamba, donde se constata la existencia de áreas cubiertas de restos cerámicos de las distintas fases cañaris, pero que, a partir de la dominación inka, «seguramente bajo la dirección de maestros cuzqueños tendería a la reproducción de la vajilla incaica con exclusividad» (Idrovo, 1990: 22); hipótesis que refuerza, con el planteamiento de Gallegos, la idea de que los alfareros «no son tan naturales», sino «traspuestos», para incrementar la producción. Por lo que subsiste la duda en torno al uso de las huactanas por parte de los alfareros mencionados en esta cita.

San Miguel de Porotos fue un sector que contaba con gran cantidad de arcilla y una importante producción alfarera al momento de la colonización española. Incluso en la actualidad todas las materias primas se obtienen de minas de la localidad. Todos los artefactos mencionados por Gallegos se utilizaban en la época de la Colonia, dato que corrobora Diego Arteaga al confrontar la Relación de Azogues con el estudio de Jozef Buys, quien expone la tipología y utilidad de cerámica presente en Cuenca en esta época: «tinajas de hacer chicha, platos, platoncillos 
medianos, botijas para transporte de vino y aceite, ollas de barro verde» (Arteaga, 2000: 41). Exceptuando las ollas verdes vidriadas que se reportan en Chordeleg, al Oriente de Cuenca, el resto todavía se produce en Jatumpamba. El documento también habla de «loza colorada», lo que concuerda con la decoración que realizan en Jatumpamba, untando una pasta rojiza sobre la superficie de las vasijas antes de su cocción.

Gallegos menciona el pueblo de «olleros», lo que podría suponer que los fabricantes de cerámica eran en su mayoría hombres, no mujeres, como sucede en la actualidad. Ante esto, Sjöman (1992: 56) propone dos hipótesis: la primera es que para el tiempo de los inkas «la producción estuviera a cargo de especialistas hombres para luego pasar a manos de mujeres como consecuencia de la migración y desmembramiento de la sociedad indígena que empezó con la invasión española»; y la segunda, que Gallegos «simplemente pasa por alto que eran mujeres las alfareras». Sin embargo, en el Perú tenemos el ejemplo de varios pueblos Ilamados Olleros, como San Bartolomé en Piura y Santo Domingo en Lima, y en ambos casos las alfareras son todas mujeres (Ramón, 1999; 2008; 2017). Durante el trabajo de campo en 2011, pude registrar el trabajo de uno de los últimos hombres alfareros del sector, Francisco Inga en ese momento de 66 años, quien aprendió el oficio de su padre a temprana edad.

\section{ETNOGRAFÍA DE LA ZONA DE ESTUDIO}

En etnografía, el trabajo de campo es un recurso utilizado desde los antropólogos clásicos. Permite obtener información directamente de los detentores del conocimiento tradicional, en este caso los alfareros de las zonas de estudio. Sin embargo, el investigador no puede irrumpir en los territorios ni actuar como autoridad etnográfica (Clifford, 1988: 53-54); debe basar su trabajo en la ética profesional. Es imprescindible establecer una relación basada en el respeto mutuo con los actores locales, exponerles los objetivos de la investigación y el nivel de colaboración que requiere para alcanzarlos. Debe comprometerse también a entregar a la comunidad los resultados de la investigación.

Al hablar de los actores locales, no me refiero exclusivamente a los detentores de los conocimientos tradicionales, sino que incluyo a las autoridades de los territorios, en este caso, a los representantes de los Gobiernos Autónomos Descentralizados de las comunidades de estudio. En coordinación con las autoridades locales planificamos varias reuniones con alfareras y alfareros; coordinamos también las fechas de las visitas a sus talleres para realizar el registro de los procesos y las entrevistas.

En Jatumpamba trabajamos con la señora Margarita Fernández, por ser la alfarera más activa de todo el sector y con el señor Francisco Inga, único hombre que todavía trabaja en cerámica. En Las Nieves, nuestros informantes fueron dos parejas de ceramistas: el señor Gregorio Morocho, su esposa Celia Ramón, Julio Ramón - hermano de Celia - y su esposa Rosa Morocho, hermana de Gregorio. Un hecho significativo, y no de poca importancia, es que en Las Nieves la actividad 
se realiza en pareja, lo que no sucede en Jatumpamba, donde es una actividad personal, como se verá más adelante.

Ya en el campo, por la naturaleza de la investigación, dos fueron las técnicas seleccionadas: la observación (participante y no participante) y la entrevista abierta (sin cuestionarios previos), para ulteriormente analizar, con el soporte del método comparativo, en los patrones de convergencia y divergencia de las tecnologías cerámicas de las dos localidades. La observación participante era fundamental para presenciar los procesos y actividades relacionadas con ellos. La entrevista, que es «la técnica privilegiada del trabajo etnográfico, pues nos permite acercarnos a las tramas de sentido, a los significados y significaciones de la acción social» (Guerrero et al., 2016: 248), fue utilizada tanto para el registro como para poder conocer y comprender el sentir de los alfareros frente a la actividad. Conforme los alfareros trabajaban, las preguntas surgían de la observación, por lo que, en ningún caso se aplicaron encuestas o entrevistas estructuradas. Para registrar las cadenas operativas utilicé cámaras de video, fotográficas, libreta de campo y grabadora de audio; y me acoplé a los tiempos que dedican los alfareros a cada actividad, por lo que el horario de las visitas estaba marcado por el avance de los procesos.

El trabajo de campo se realizó durante dos meses de manera simultánea en las dos localidades, entre mayo y julio de 2011. Para detectar las tramas de significado que esta actividad tiene en las comunidades, las entrevistas abarcaron un espectro más amplio de población. Así, en Jatumpamba se entrevistaron a doce personas y en Las Nieves a seis, todos artesanos activos.

\section{TECNOLOGÍA CERÁMICA EN JATUMPAMBA}

A continuación, se detallará el proceso de elaboración de las ollas de Margarita Fernández que, al igual que las otras alfareras de Jatumpamba, produce también tiestos (igualmente conocidos como torteros, tostadoras o callanas), cántaros y macetas que comercializa a intermediarios que acuden a su taller. Generalmente, fabrica sus vasijas en grupos de diez o quince de manera simultánea.

Todas las materias primas — barro, arena y la tierra roja para decoración (quina)_ se obtienen en Jatumpamba, Pacchapamba y Chico Ingapirca y también en zonas aledañas como San Cristóbal y Olleros. Los materiales son extraídos con palas, picos y lampas, y transportados hacia los talleres y cargados sobre las espaldas de las señoras en saquillos o canastos de mimbre.

De la mina al taller el barro llega troceado en pedazos grandes. Para poder triturarlo, tiene que estar completamente seco,/; caso contrario, al momento de hidratarlo, no absorbe el agua de manera uniforme. Margarita lo hace en una acera de la casa (fig. 6a). La herramienta para triturar suele ser un mazo de madera o un tubo metálico. Cuando los terrones han reducido su tamaño, los golpea directamente en el piso hasta pulverizarlos.

Al tener la cantidad suficiente, recoge el barro en una cesta de mimbre y lo vierte en un recipiente de plástico con capacidad de veinte kilogramos, al que agrega 
agua hasta que rebose unos centímetros. En dos días el barro ya ha absorbido toda el agua y está totalmente hidratado. La mezcla con el desgrasante se realiza en un lugar cubierto y con piso de tierra. Margarita esparce homogéneamente la arena para que el barro no se pegue al piso. Vierte el barro del recipiente sobre esta base de arena y con sus manos forma un cúmulo en cuyo centro hace un pequeño boquete sobre el que arroja arena con sus manos; a nuestro juicio, la proporción es equivalente a un tercio de arena por dos tercios de barro.

Comienza a pisar y a formar la mezcla con su pie derecho (fig. 6b). Al poco tiempo, con sus dos pies pisa la pasta hasta que se expanda hacia afuera. Después, desde el centro, con sus manos junta la pasta hasta volver a tener un cúmulo con el que inicia la labor nuevamente. Este proceso se repite varias veces. En ciertos momentos agrega pequeñas cantidades de agua a la pasta. Margarita sabe que la pasta está lista cuando «ya se envuelve nomás solita», lo que, en mi interpretación, significa que la pasta es homogénea.

La alfarera separa la pasta en varias porciones a las que llama «bolitas», que se transformarán, cada una, en una olla. Coloca una porción sobre la base de una vieja tinaja de unos $70 \mathrm{~cm}$ de altura que se encuentra boca abajo en el patio de su casa desde hace ya varios años, a la que previamente esparce arena como antiadherente: «si no pongo arena entonces después no se levanta, se pega». Con

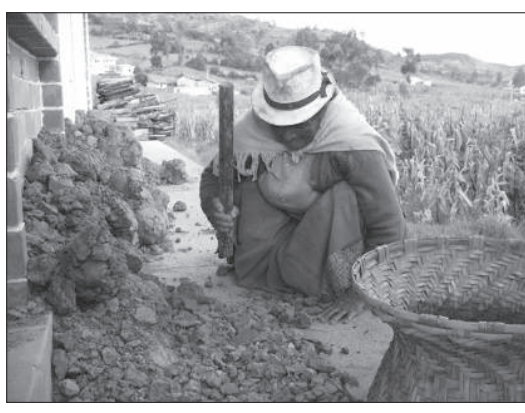

(a) Troceado y triturado del barro

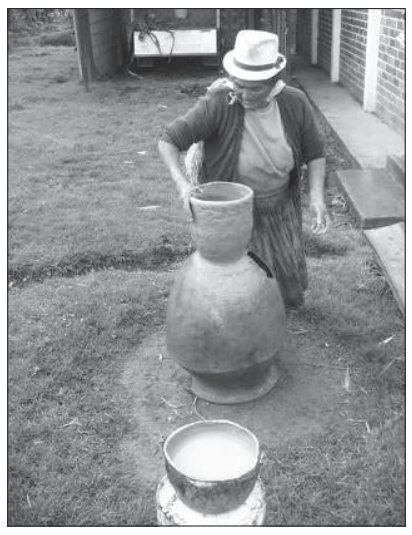

(c) Modelado y decorado del borde

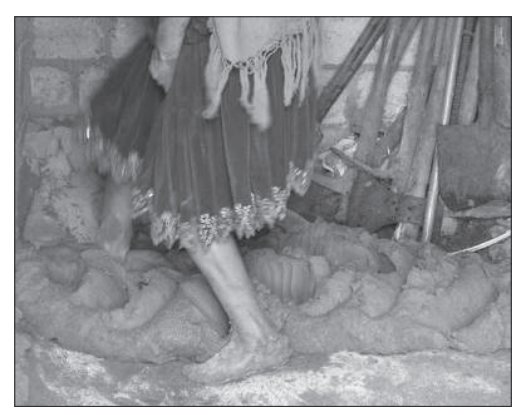

(b) Mezcla de barro con desgrasante

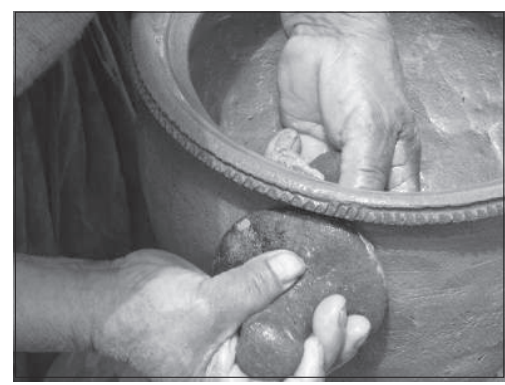

(d) Primer golpeado: formación y primer alisado del cuello 


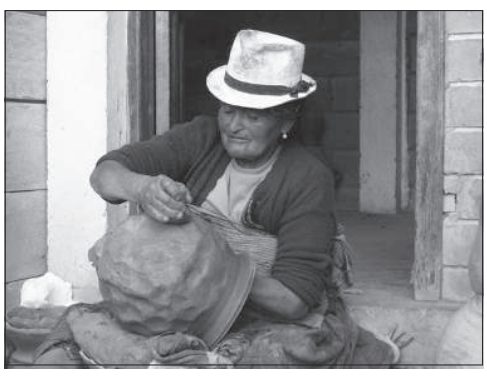

(e) Segundo golpeado: creación del cuerpo globular

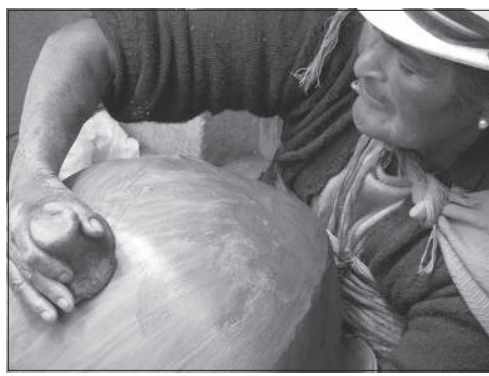

(g) Alisado del cuerpo globular, creación de la base

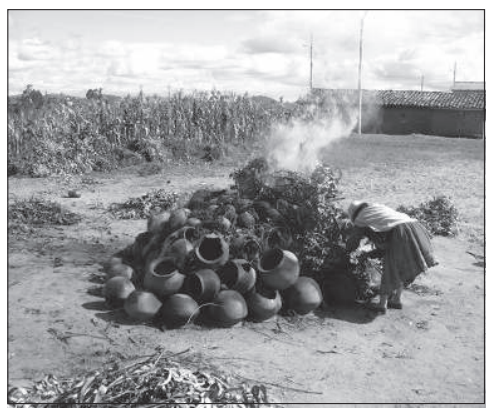

(i) Cocción en estructura de quema a cielo abierto

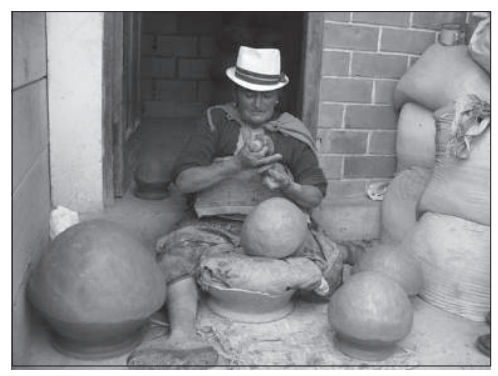

(f) Tercer golpeado: unificación de las paredes del cuerpo globular

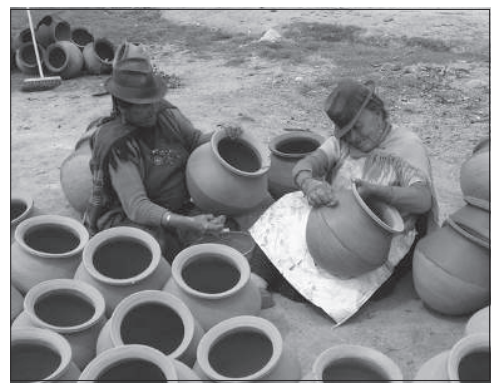

(h) Engobe del borde y del cuello de la olla

Figura 6 - Cadena operativa de Jatumpamba

(c) M. Brazzero, 2011

la base de la tinaja como soporte, modela la pasta con las manos húmedas hasta formar un cilindro compacto. Introduce el puño de su mano derecha desde arriba hasta la mitad del cilindro, mientras que con la otra sostiene las paredes desde afuera. Con los dedos de la mano derecha, estira la reserva de pasta desde el interior hacia arriba y hacia afuera mientras gira su cuerpo alrededor de la pieza que va formando. Es acertada la apreciación de Sjöman (1992: 65) que dice que las alfareras de Jatumpamba son «su propio torno» ya que, en el proceso, quien gira es la alfarera, no la cerámica. Al llegar a la altura deseada comienza a formar el borde de la olla (fig. 6c). Para esto se sirve de un pequeño cuero mojado que 
sostiene con su mano derecha: el pulgar presiona desde adentro, y los otros dedos actúan desde afuera mientras gira su cuerpo hacia su izquierda, alrededor de la olla. Después, hace un movimiento similar, pero con sus dos manos: la izquierda por dentro y la derecha por fuera del borde hasta adquirir una forma evertida. Con el cuero doblado hace unas pequeñas impresiones verticales con su mano derecha en el borde que llama «crespitas», girando esta vez hacia su derecha. Estas impresiones y, en ocasiones una línea horizontal en todo el perímetro del borde, más el engobe, constituyen la única decoración de las ollas. Terminado el borde, retira la pieza de la tinaja y la pone a secar a la sombra hasta el día siguiente para que adquiera firmeza.

Al día siguiente, Margarita coloca la olla sobre la base de la tinaja para formar el cuello. Aquí, entran en escena las huactanas o golpeadores que, como se dijo, son de dos tipos: una cóncava usada para golpear la olla por fuera, y otra convexa para trabajar por dentro de la olla. Con la huactana convexa, que funciona como yunque, sostiene con su mano derecha la pared interna, y con la izquierda golpea la pared externa utilizando la huactana cóncava para dar forma al cuello o huigsanchir (fig. 6d). Este tratamiento deja las paredes irregulares. Para volverlas uniformes, la alfarera gira alrededor de la olla presionando de a poco las paredes interna y externa, con las huactanas siempre humedecidas. Con esta fricción, modela y alisa la parte superior, controlando su grosor y cubriendo imperfecciones. Una vez que ha obtenido un cuello liso e inclinado hacia afuera en la sección media de la olla, detiene el proceso y termina la jornada.

Para el siguiente paso la alfarera ya no utiliza la tinaja invertida, trabaja sentada en el suelo sobre una estera, en el portal de su casa. A su derecha, ubica un recipiente con agua y las huactanas. Entre sus piernas, pero asentado sobre la estera, elabora un pedestal con el cuello invertido de una olla rota, sobre el que coloca telas viejas para lograr una superficie blanda donde asienta la olla en posición inclinada. Inicia el segundo golpeado humedeciendo las huactanas para trabajar el segmento de la olla que Holm (1961) Ilama «cuerpo globular» y Margarita «pecho y cintura» que, en este momento del proceso, es muy maleable. La alfarera sostiene el golpeador convexo en el interior del cuerpo globular como yunque, mientras que, con el cóncavo, golpea la pared exterior para extender el barro, consiguiendo una forma ovoide todavía bastante rústica, pero respetando el borde y el cuello que fueron terminados en la jornada anterior (fig. 6e).

De manera similar al paso anterior, a la mañana siguiente, la alfarera toma una huactana en cada mano para terminar el cuerpo globular, pero esta vez logra una forma redondeada, unificando el grosor de las paredes. Cuando obtiene la forma adecuada, que responde a una altura superior a la línea media de la olla, la deja secar por un lapso de 2 a 3 horas antes de iniciar el proceso de alisado (fig. 6f). Con la huactana cóncava, siempre humedecida, alisa el exterior, y con la huactana convexa el interior, girando la olla en el pedestal; después, la coloca boca abajo directamente sobre el suelo, y, con movimientos circulares de la huactana convexa, crea la base para que la olla pueda sostenerse sola al ponerla en pie (fig. 6g). 
Incluyendo los tiempos de secado, la elaboración de una serie de diez vasijas, previo a la aplicación del engobe y a la cocción, tarda entre cinco a ocho días, dependiendo de las condiciones del clima, en particular de la humedad ambiental. El engobe, único efecto cromático, se realiza en la mañana del día de la cocción; todas las ollas deben estar secas. Las dos ocasiones que estuve presente, se quemaron 100 y 160 respectivamente. Margarita contrata a ayudantes, generalmente mujeres de la comunidad, pues se necesita manos para el arduo proceso. Una de las ayudantes prepara el engobe en un recipiente de aluminio mezclando tierra roja (quina) y agua hasta obtener una consistencia pastosa. Enseguida, con una tela aplica el engobe en el borde y cuello por fuera de la olla, y por dentro solo lo aplica en el borde interno (fig. 6h). En ningún caso vi que pintaran el cuerpo globular. Una vez aplicada, la quina está totalmente seca en unas dos a tres horas, y la vasija se encuentra lista para la cocción.

El lugar elegido para la quema a cielo abierto es una explanada de tierra a pocos metros de la casa de Margarita, frente a la capilla de Pacchapamba. Las condiciones necesarias para iniciar la cocción son muy especiales: el día tiene que ser soleado y ventoso para que se mantenga el fuego. Se inicia colocando las ollas grandes y los cántaros en la base de lo que será la estructura de quema (localmente llamada «horno»), asentadas de costado sobre pequeños fragmentos de ollas rotas.

Margarita ubica cien ollas y cántaros alternados en hileras de diez, adosados boca con base, creando una plataforma cuadrada, sobre la cual tiende una capa de leños de eucalipto. Sobre esta capa de leña coloca unas sesenta ollas medianas siguiendo el mismo orden que para el primer grupo, pero dejando un perímetro externo. Dispone nuevamente leños y termina con una última capa de tiestos y ollas pequeñas, pero dispuestos boca abajo. La apariencia final de la estructura de quema es de una pirámide escalonada de planta cuadrada. Finaliza poniendo gran cantidad de leña de eucalipto y chamiza (leña menuda recolectada en los cerros del sector) en tres de los costados de la estructura de quema, que sostiene con ollas rotas o mal quemadas.

Para encender la estructura de quema, Margarita recoge brazas ardientes con un fragmento de olla de una hoguera que encendió a pocos metros, y las desplaza hacia la chamiza, por debajo de la leña. Una vez que se enciende el fuego, agrega una y otra vez chamiza seca y ramas de eucalipto (fig. 6i).

Unos treinta minutos más tarde, las ollas — que se tornaron negras desde el inicio de la quema-, poco a poco se van oxidando, y tomar el color rojo-anaranjado característico de esta cerámica. Este es el momento de trasladar el fuego al otro costado de la estructura de quema, que no estuvo en contacto directo con la llama. Una vez consumido el fuego, y cuando las últimas vasijas cobran el color esperado, se deja enfriar la estructura de quema; tres horas después, se la desmantela, retirando primero las ollas rotas que sujetaron la chamiza. En total, la quema tarda aproximadamente cinco horas. Con la ayuda de varas de madera, los artefactos recién quemados se esparcen en el piso de tierra hasta que se hayan enfriado. Ya entrada la tarde, se los guarda en una bodega para que esperen el día de su viaje al mercado. 


\section{TECNOLOGÍA CERÁMICA EN LAS NIEVES}

La parroquia Las Nieves tiene minas de dos tipos de barro, el negro y el blanco (este último llamado suco), extraídos de la colina Jachapamba, ubicada a dos horas de caminata de la cabecera parroquial. Estas denominaciones de «negro y suco» se deben a la evidente diferencia de color de las tierras. El barro suco es más arenoso, por lo que es utilizado como desgrasante. Los hombres extraen el material con picos, palas y barretas y las mujeres lo llenan en saquillos que son transportados hasta los talleres cargados por caballos de su propiedad. La mina de quina se encuentra en la parroquia Cochapata del cantón Nabón, ubicada a unos 33 kilómetros de distancia. Trasladan la quina en camionetas (lo que les tarda unas dos horas, entre ida y vuelta) o a caballo (alrededor de cuatro horas). Los tipos de cerámica que se fabrican son: ollas, cántaros, tiestos, tinajas, maceteros.

Los talleres, en los casos registrados, están ubicados en la planta baja de las viviendas, que son de dos plantas, y donde una habitación se destina únicamente a actividades de alfarería. Celia y Julio, por separado, trituran la arcilla en el portal de la casa. Extienden directamente el barro seco sobre un plástico y lo golpean con un mazo de madera para después poder recogerlo fácilmente (fig. 7a). Celia mezcla los dos tipos de arcilla, la negra y la suca, en proporciones de tres a dos aproximadamente; luego, abre un boquete en el que vierte agua y, con los dos pies, pisa la pasta extendiéndola sobre el plástico. Esta actividad se repite varias veces hasta que la pasta deje de pegarse al plástico y se torne homogénea. Finalmente, envuelve la pasta con el plástico para mantener la humedad (fig. 7b).

El proceso se realiza en pareja. Celia y Gregorio trabajan directamente sobre una mesa en la habitación que funciona también como bodega de cerámica. Gregorio toma una porción de pasta y la modela hasta conseguir una forma semiesférica que la mejora, asentándola sobre la mesa. Monta la pasta modelada sobre un soporte que no es nada más que el borde y cuello invertido de una olla descartada, de la cual se ha eliminado previamente los excesos de cerámica con pequeños golpes de martillo (fig. 7c).

A continuación, Celia continúa la tarea iniciada por Gregorio. Sobre esta base, la nueva olla va tomando forma al incorporar sucesivos cordeles gruesos de pasta húmeda, alargados y cilíndricos, que se adhieren a las paredes desde el interior (fig. 7d). Con una mano, Celia une el cordel a la base, lo aplana y extiende desde el interior y hacia arriba, cuidando la forma exterior con la otra mano. Rectifica las paredes incorporando trozos de pasta en aquellos lugares menos gruesos o con imperfecciones. Conforme coloca nuevos cordeles crecen las paredes de la olla hasta alcanzar unos $40 \mathrm{~cm}$; entonces, con cordeles cada vez más cortos, va disminuyendo el diámetro de la olla. Los últimos cordeles se agregan desde afuera de las paredes. Es importante anotar que los alfareros trabajan de pie y giran el pedestal de cerámica cocida durante todo el proceso, mientras construyen la vasija.

Seguidamente, Celia alisa las paredes, tanto por fuera como por dentro, y fabrica el cuello y el borde; utiliza el golpeador convexo humedecido para evertir el borde con movimientos circulares desde el interior, hasta lograr un cuello alisado que termina en un borde de menor diámetro que el cuerpo (fig. 7e). 
Austro ecuatoriano: etnografía comparada de procesos de elaboración de cerámica en dos comunidades

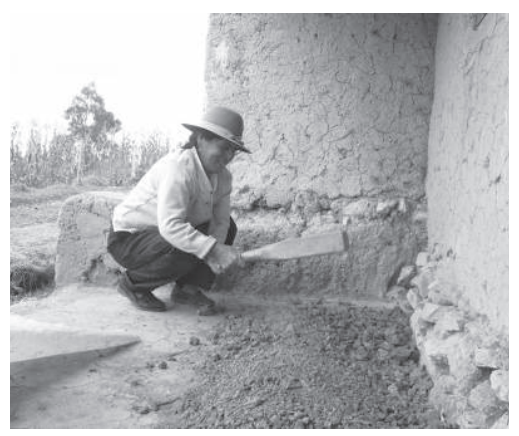

(a) Troceado y triturado del barro

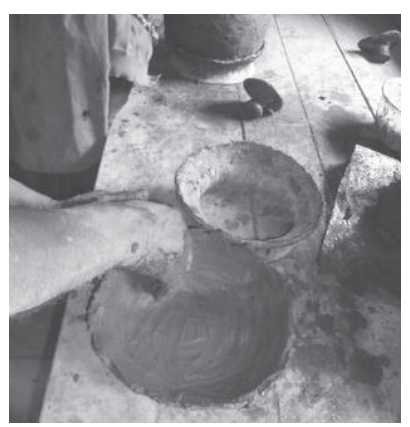

(c) Formación de la base: modelado y molde

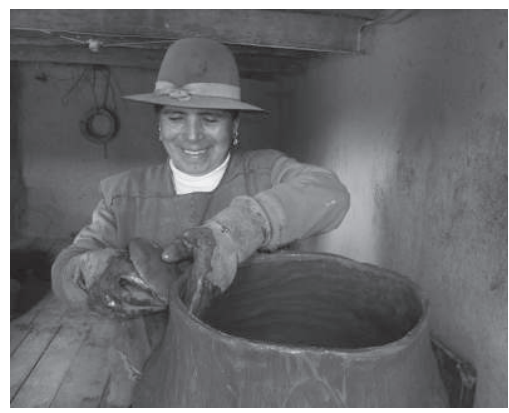

(e) Golpeado del cuerpo de toda la olla

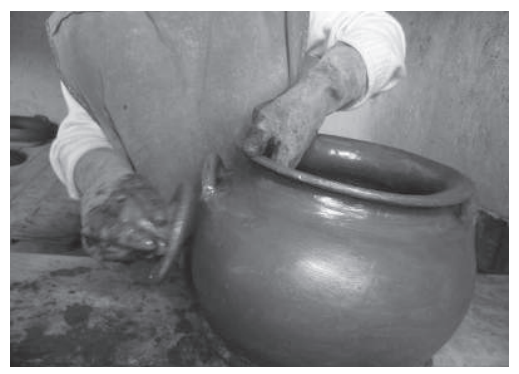

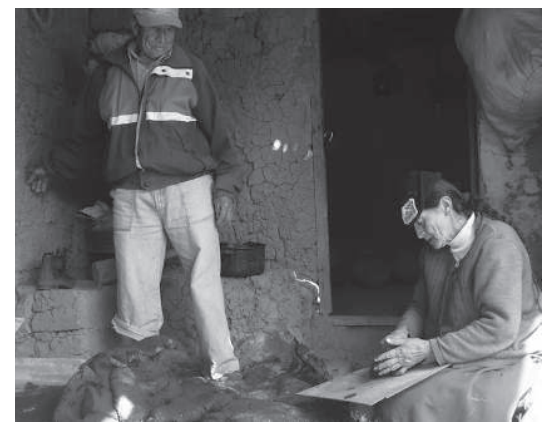

(b) Mezcla de barro con desgrasante

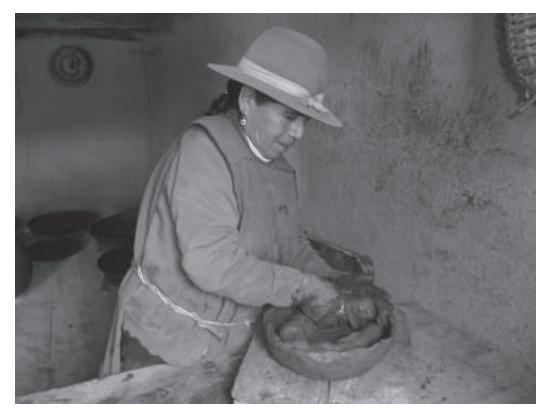

(d) Formación de las paredes con cordeles

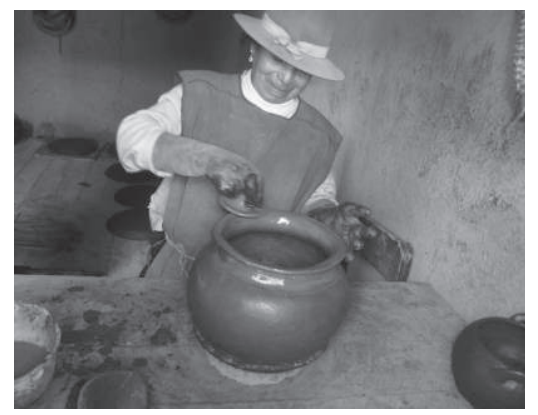

(f) Primer alisado y formación de borde

(g) Segundo alisado 


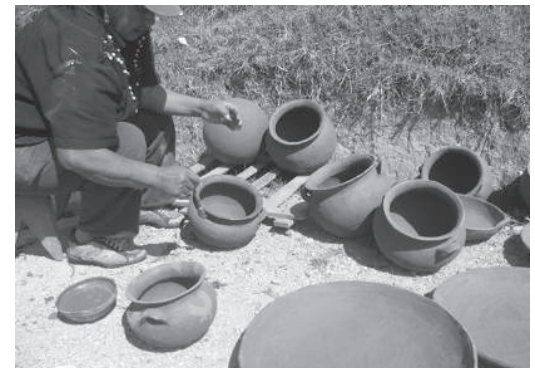

(h) Decoración

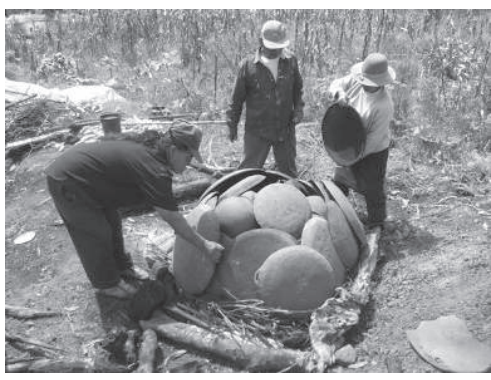

(i) Cocción

Figura 7 - Cadena operativa de Las Nieves

(C) M. Brazzero, 2011

En la jornada siguiente, Celia utiliza los golpeadores para expandir la olla, haciendo girar repetidamente el pedestal de barro cocido sobre la mesa de trabajo. Sin tocar el cuello, golpea las paredes extendiendo su contorno en la zona media, hasta lograr una forma relativamente esférica. A continuación, con el golpeador convexo humedecido, alisa las paredes del cuello, cuidando de no deformarlo, y crea el borde (fig. 7f). Celia finaliza colocando dos asas que llama «orejas» en los lados opuestos de la olla, a la misma altura y en sentido horizontal, con dos pequeños cordeles de barro, a los que da una curvatura en forma de herradura. Los adhiere extendiendo los bordes con las manos humedecidas hasta perderlos en las paredes de la olla (fig. $7 \mathrm{~g}$ ).

Al día siguiente, Celia retira la olla del soporte y la pasa a Gregorio. Sentado en el portal de la casa, él la apoya invertida en sus piernas para alisar las imperfecciones propias del contacto con el soporte y formar la base con el golpeador cóncavo humedecido, para que pueda autosustentarse.

La elaboración de ocho vasijas y su secado, previo a la aplicación de la decoración y la quema, varía entre cinco y siete días, tiempo que depende (al igual que en Jatumpamba) de las condiciones del clima.

El día de la quema debe estar soleado y ventoso. Por la mañana, las mujeres decoran las ollas que deben ya estar secas. Celia utiliza una tierra roja extraída de minas ubicadas en la parroquia Cochapata. La humedece hasta lograr una consistencia pastosa que aplica sobre las ollas con un palo pequeño que tiene una tela envuelta en la punta, a manera de hisopo, con el que imprime líneas oblicuas como única decoración (fig. 7h). A diferencia de Jatumpamba, en Las Nieves solamente aplican el engobe para decorar con líneas las paredes y el borde.

La estructura de quema se fabrica con las ollas de las dos parejas de alfareros, en un espacio abierto a pocos metros de sus casas, ya que, además de ser familiares, son vecinos. Sobre una base de leña delgada que llaman «colchoncito», colocan las ollas más grandes, unas junto a otras, formando una planta circular. Sobre ellas van las ollas medianas que cubren con tiestos invertidos, formando una especie de caparazón (fig. 7i). 
Los hombres cubren esta estructura con tiestos dañados de quemas anteriores desde el piso, de manera que apenas se puede ver hacia el interior. En los espacios que quedan entre estos tiestos, introducen hojas de penco secas y leños delgados de eucalipto, nogal, chilca y chahuarquero (palo mayor del penco). Leños gruesos de eucalipto se colocan en la base, por todo el perímetro de la estructura de quema, sobre los que acumulan leños pequeños adosados a las paredes exteriores.

La estructura de quema se enciende desde un orificio que se dejó en la base y que alimentan con hojas secas de eucalipto hasta que prenda por completo. Las dos parejas agregan hojas de penco y chamiza por encima y por los lados de la estructura de quema de manera periódica. Los hombres utilizan horquetas de madera para acomodar los leños y la chamiza. Ollas y tiestos están listos cuando han cambiado su color, «ya no están negros, están amarillos»; el tiempo transcurrido desde que inició la quema es de tres horas aproximadamente.

En un espacio abierto, a pocos pasos de la estructura de quema, Rosa prepara el suelo barriendo el piso con un atado de ramas para eliminar cualquier hoja que pueda encenderse con el calor de los objetos recién retirados de la estructura de quema. Si se encienden estas hojas, «negrean y manchan las ollas».

Con la horqueta remojada en agua, Julio retira primero los restos de troncos y los tiestos viejos. Después, los tiestos y ollas ya quemados se ubican en el espacio preparado por Rosa. En esta estructura, se queman alrededor de sesenta artefactos. Es mucho más pequeña que las de Jatumpamba, lo que hace que la labor no sea tan agobiante.

\section{CONCLUSIONES}

De manera general, los pocos estudios arqueológicos existentes no toman en cuenta la dimensión tecnológica de manera profundizada. No son concluyentes al establecer el origen Tacalshapa de la técnica del golpeado; tampoco establecen con rigurosidad una continuidad entre el uso prehispánico y actual de los golpeadores. Los artefactos analizados por Holm pertenecían a museos y colecciones particulares, pero no contaban con información del contexto arqueológico de origen. La atribución de Sjöman e Idrovo a la fase Tacalshapa se sustenta en un único golpeador reportado en el contexto Cañari-Inka del sitio Pumapungo, sobre el que no dan mayores detalles; tampoco explican las tecnologías cerámicas de la mencionada fase. Sin embargo, a nivel regional, trabajos más recientes que abordan la tecnología cerámica aplicando la comparación entre los datos etnográficos y el material arqueológico, realizados por Catherine Lara (2019), demuestran el uso del golpeador desde Tacalshapa I hasta Cashaloma.

En este sentido, las investigaciones sobre el enfoque tecnológico; que identifica las cadenas operativas, como los realizados por Lara $(2014 ; 2017$; 2019) son imperativas. Conocer los materiales empleados, la pasta y desgrasante, las prácticas sociales en torno a la producción y la comercialización, entre otros aspectos, nos brindaría un mayor horizonte de análisis teórico. Para poder comprobar el uso de 
la huactana desde tiempos prehispánicos en Jatumpamba y en Las Nieves, habría que aplicar métodos similares a materiales arqueológicos excavados en estas localidades. De hecho, llama la atención que no existan excavaciones científicas en zonas con presencia etnográfica de golpeadores, por ejemplo.

$\mathrm{Si}$ bien las cadenas operativas en las zonas de estudio tienen elementos comunes, como el uso de los golpeadores y la quema a cielo abierto, difieren en ambos casos (cuadro 1). Mientras que en Jatumpamba usan el modelado/golpeado, en Las Nieves utilizan el moldeado/acordelado/golpeado. La constatación de estas diferencias durante el trabajo de campo; me lleva a plantear las siguientes preguntas: ¿pudo darse una difusión de las herramientas de trabajo - los golpeadores- hacia varios sectores de la Sierra Sur del Ecuador que, incorporándolos a su producción, mantuvieron sin embargo sus tradiciones técnicas locales (Lara, 2019). Si a través de contactos culturales se pudieron difundir los golpeadores, mas no las tradiciones técnicas, ¿cuál fue el centro desde el cual se irradió el uso de estas herramientas y bajo qué modalidad de contacto?

El registro etnográfico realizado por quien suscribe (Brazzero, 2011) en San Miguel y Nabón; y por Lara (2017) en San Miguel, Sígsig, Nabón y Taquil (fig. 1) corrobora las diferencias en las tradiciones técnicas locales y en el uso de los golpeadores, pero la forma de difusión de estas herramientas, como de los antiguos circuitos de comercio de los artefactos cerámicos dentro del territorio, son temas pendientes. Sin embargo, se puede asegurar que la presencia de estas herramientas en distintas áreas y contextos culturales de las provincias de Cañar, Azuay y Loja, no puede ser fruto de la casualidad ni de paralelismos tecnológicos.

Poco a poco vemos que la alfarería con técnicas ancestrales se va perdiendo. Las razones son varias. Los testimonios hablan de lo escasas que se vuelven la madera y la chamiza para la cocción en los bosques colindantes. El precio de la leña no representaría una inversión favorable con relación al costo de venta de las vasijas, tomando en cuenta que, en los dos casos, se comercializan con intermediarios. Ante la preferencia de utensilios de aluminio, de hierro o cerámica industrial en los centros poblados, el turismo no representa una alternativa, debido al tamaño y fragilidad de la cerámica que, difícilmente, la convierte en un souvenir. Las nuevas generaciones no desean aprender el oficio debido a que los beneficios económicos no compensan el esfuerzo de producción. Pero también hay una razón subjetiva: los jóvenes tienen otras expectativas de vida y desvalorizan estas prácticas. Su atención se centra en migrar fuera del país o salir a trabajar en ciudades como Cuenca, Azogues o Guayaquil. «Ya no quieren ensuciarse las manos con el barro, prefieren lucir como la gente de la ciudad», me comenta Carmen, joven habitante de Jatumpamba, hija de Margarita.

El involucramiento de las universidades, museos e instituciones culturales es fundamental. Pienso en varios aspectos: prospecciones arqueológicas en los lugares que reportan cerámica etnográfica; trabajo interdisciplinario para desarrollar proyectos etnoaqueológicos que contextualicen, describan las formas, técnicas e instrumentos utilizados; implementación de laboratorios para realizar análisis químicos de las piezas etnográficas y arqueológicas, proyectos de investigación colaborativos que permitan generar protocolos de comparación de resultados. 
Austro ecuatoriano: etnografía comparada de procesos de elaboración de cerámica en dos comunidades

Cuadro 1 - Cuadro comparativo de las cadenas operativas en las zonas de estudio

\begin{tabular}{|l|l|}
\hline CADENA OPERATIVA EN JATUMPAMBA & CADENA OPERATIVA EN LAS NIEVES \\
\hline $\begin{array}{l}\text { TIPOLOGÍA DE LA CERÁMICA } \\
\text { Ollas, vasijas, piñatas, tiestos, cántaros, } \\
\text { macetas }\end{array}$ & $\begin{array}{l}\text { TIPOLOGíA DE LA CERÁMICA } \\
\text { Ollas, cántaros, tiestos, tinajas, macetas }\end{array}$ \\
\hline Recolección de materia prima & Recolección de materia prima \\
\hline Troceado y triturado del barro & Troceado y triturado del barro \\
\hline Mezcla de barro con desgrasante & Mezcla de barro con desgrasante \\
\hline Pisado para unificar la pasta & Pisado para unificar la pasta \\
\hline Modelado del borde y el cuello & Formación de la base: modelado y molde \\
\hline $\begin{array}{l}\text { Primer golpeado: formación y primer } \\
\text { alisado (o «pulido») del cuello }\end{array}$ & Formación de las paredes con cordeles \\
\hline $\begin{array}{l}\text { Segundo golpeado: creación del cuerpo } \\
\text { globular }\end{array}$ & Golpeado del cuerpo de toda la olla \\
\hline $\begin{array}{l}\text { Tercer golpeado: unificación de las } \\
\text { paredes del cuerpo globular }\end{array}$ & Primer alisado y formación de borde \\
\hline $\begin{array}{l}\text { Alisado del cuerpo globular y creación de } \\
\text { la base }\end{array}$ & Segundo alisado \\
\hline Engobe del borde y cuello de la olla & Decoración \\
\hline $\begin{array}{l}\text { Cocción en estructura de quema a cielo } \\
\text { abierto }\end{array}$ & $\begin{array}{l}\text { Cocción en estructura de quema a cielo } \\
\text { abierto }\end{array}$ \\
\hline
\end{tabular}

(C) Elaboración propia

En conjunto, estos campos de investigación pueden constituirse en aportes científicos que, junto a los horizontes estilísticos, fortalezcan sustancialmente los planteamientos sobre la arqueología regional; y que, como requiere este caso, ratifiquen o descarten posibles contactos culturales y relaciones interregionales entre las tecnologías cerámicas que perviven en zonas alfareras de la región cañari, entre ellas la técnica del golpeado.

\section{Referencias citadas}

ARTEAGA MATUTE, D., 2000 - El Artesano en la Cuenca Colonial, 1557-1670, 175 pp.; Cuenca: Casa de la Cultura Ecuatoriana-Núcleo del Azuay, Centro Interamericano de Artesanías y Artes Populares-CIDAP.

BRAZZERO, M., 2011 - Investigación del patrimonio cultural inmaterial vinculado a la alfarería con técnicas prehispánicas de Jatunpamba y Las Nieves, 89 pp.; Cuenca. Informe presentado al INPC.

CLIFFORD, J., 1988 - Dilemas de la Cultura: antropología, literatura y arte en la perspectiva posmoderna, 429 pp.; Barcelona: Ed. Gedisa. 
COLLIER, D. \& MURRA, J. V., 2007 [1943] - Reconocimiento y Excavaciones en el Austro Ecuatoriano, 465 pp.; Cuenca: Casa de la Cultura Ecuatoriana, Núcleo del Azuay.

GALLEGOS, G. de, 1897 [1582] - San Francisco de Peleusi del Azogue. In: Relaciones Geográficas de Indias, Tomo III: 274-278; Perú, Madrid: Ediciones Atlas.

GOMIS, D., 2007 - El Territorio Austral Durante el Formativo Tardío: Apéndice C. In: Reconocimiento y excavaciones en el Austro Ecuatoriano (D. Collier \& J. V. Murra, eds.): 291-328; Cuenca: Casa de la Cultura Ecuatoriana, Núcleo del Azuay.

GOMIS S., D., 2019 - Max Uhle y el tiempo en la América Andina. Evolucionismo, difusionismo y medición del tiempo relativo. In: Max Uhle, aportes a la arqueología del austro ecuatoriano (T. Landivar Villagómez, J. Idrovo Urigüen, M. Sempértegui Cárdenas, M. Guiracocha Llivipuma, eds.): 31-37; Cuenca: Museo y Parque Arqueológico Pumapungo, Universidad del Azuay.

GUERRERO, P., FERRARO, E. \& HERMOSA, H., 2016 - El trabajo antropológico. Miradas teóricas, metodológicas, etnográficas y experiencias desde la vida, 544 pp.; Quito: Ediciones Abya Yala, Universidad Politécnica Salesiana.

HOLM, O., 1961 - La Técnica Alfarera de Jatumpamba (Ecuador). Cuadernos de Historia y Arqueología, N. ${ }^{\circ}$ 27: 153-210.

IDROVO URIGËN, J., 1990 - Siglos XVI y XVII: la desarticulación del mundo andino y sus repercusiones en la alfarería indígena del Austro Ecuatoriano. In: Cerámica Colonial y Vida Cotidiana (J. Idrovo Urigüen \& A. Kennedy Troya, eds.): 21-38; Cuenca: Fundación Paul Rivet.

IDROVO URIGËN, J., 2000 - Tomebamba, Arqueología e Historia de una Ciudad Imperial, 348 pp.; Cuenca: Banco Central del Ecuador Regional Cuenca.

LARA, C., 2014 - Tecnología Cerámica y Ocupación Precolombina de Las Estribaciones Andinas: El Caso Del Valle Del Río Cuyes. Apuntes: Ecuador, Arqueología Y Diplomacia. Consulta: 15-10-15. Disponible en: http://arqueologia-diplomaciaecuador.blogspot.com/2014/07/tecnologia-ceramica-y-ocupacion.html (Consulta el 15 de mayo de 2011).

LARA, C., 2017 - Aportes del enfoque tecnológico a la arqueología precolombina: pasado y presente de la alfarería en el valle del río Cuyes y su región (Andes sur-orientales del Ecuador), 248 pp.; Oxford: Archaeopress Publishing.

LARA, C., 2019 - Tacalshapa y Cashaloma: perspectivas del enfoque tecnológico. Revista de Historia, Patrimonio, Arqueología y Antropología Americana, N. ${ }^{0}$ 1: 180-199.

MEYERS, A., 1998 - La Tradición Tacalshapa y la Arqueología del Cañar y Azuay en la Sierra Sur del Ecuador: Una Secuencia a base de Comparaciones con el Norte del Perú: 169-198; Bonn-Alemania: Verlag Anton Saurwetn.

RAMÓN JOFFRÉ, G., 1999 - Producción alfarera en Santo Domingo de los Olleros (Huarochirí- Lima). Bulletin de l'Institut Français d'Études Andines, 28 (2): 215-248. https://www.researchgate.net/publication/26430896_Produccion_alfarera_en_ Santo_Domingo_de_los_Olleros_Huarochiri__Lima/citations (Consulta ê $\overline{0} \overline{1}$ de junio de 2020).

RAMÓN JOFFRÉ, G., 2008 - Producción alfarera en Piura (Perú): estilos técnicos y diacronía. Bulletin de I'Institut Français d'Études Andines, 37 (3): 477-509. Publicado el 01 junio 2009, consultado el 13 julio 2020. URL: http://journals.openedition.org/ bifea/2985; DOI: https://doi.org/10.4000/bifea.2985 (Consulta el 01 de junio de 2020).

RAMÓN, G., 2017 - Los alfareros itinerantes de Cuzcudén, San Pablo, Cajamarca. IFEA. https://ifea.hypotheses.org/1016 (Consulta el 01 de junio de 2020). 
Austro ecuatoriano: etnografía comparada de procesos de elaboración de cerámica en dos comunidades

SJÖMAN, L., 1991 - Cerámica Popular, Azuay y Cañar, 121 pp.; Cuenca: Centro Interamericano de Artesanías y Artes Populares (CIDAP).

SJÖMAN, L., 1992 - Vasijas de Barro, la cerámica popular en el Ecuador, 404 pp.; Cuenca: Centro Interamericano de Artesanías y Artes Populares (CIDAP). 